tor delay, frontal bossing and temporal 'retraction', microcephaly, a large mouth with thin lips and downturned corners, a cleft or high arched palate, micrognathia, low set malformed ears, a short and/or webbed neck, a widow's peak, a low posterior hairline, widely spaced nipples, congenital heart defects, renal anomalies, rhizomelia, polydactyly, and hyperlaxity of limb joints. Several of these features were present in our case although her early death and lack of necropsy prevented further, more detailed examination being carried out. Interesting clinical findings in our case were the massive oedema, anaemia, and calcified gallstones. These features have not been described in any of the previous reports. It is possible that they may be the result of the deletion of the terminal region of $12 p$. However, there have been very few published reports of $12 p$ deletions and clinical manifestations have been non-specific with phenotypic variation probably resulting from differences in the size of the deleted segment. The unique features described in our case have not been recorded in any of the cases of $12 p$ deletion. ${ }^{4}$

Recognition of syndromes, whether chromosomal, single gene, or those of unknown aetiology, is important for early diagnosis and initiation of cytogenetic analysis, when appropriate, because of the important implications for genetic counselling, for family studies to detect others at risk, and for prenatal diagnosis. New cases with additional or unusual features are important both for the further delineation of the syndrome and to describe features which may be useful in diagnosis, whether prenatally or in the newborn period. Further follow up was not possible in our case because the parents returned abroad to their country of origin.

\footnotetext{
References

1 Bridge J, Sanger W, Mosher G, et al. Partial duplication of distal 17q. Am J Med Genet 1985;22:229-35.

2 Serotkin A, Stamberg J, Waber L. Duplication 17q mosaicism: an infant with features of Ellis-van Creveld syndrome. $J$ Med Genet 1988;25:258-60.

3 Orye E, Van Bever H. De novo distal trisomy 17q. Ann Genet (Paris) 1985;28:61-2.

${ }^{4}$ Kivlin JD, Fineman RM, Williams MS. Phenotypic variation in the del(12p) syndrome. Am J Med Genet 1985;22:769-79.
}

Correspondence to Dr A Caine, Regional Cytogenetics Unit, St James's University Hospital, Beckett Street, Leeds LS9 7TF.

\title{
Moebius' syndrome with unilateral cerebellar hypoplasia
}

\author{
M G HARBORD, J P FINN. M A HALL-CRAGGS, E M BRETT, AND \\ M B ARAITSER \\ The Hospitals for Sick Children, Great Ormond Street, London WC1N 3JH.
}

SUMMARY A case is reported of a child with Moebius' syndrome who also has unilateral cerebellar hypoplasia. We suggest that this combination of abnormalities could result from a vascular disruption occurring in the basilar artery early in its development.

Moebius' syndrome consists of congenital unilateral or bilateral facial weakness and loss of abduction of the eye, which are interpreted clinically as defects of the seventh and sixth cranial nerves. ${ }^{1}$ The involvement of other cranial nerves or abnormalities of the extremities, such as syndactyly, agenesis of digits, or defective branchial musculature, are common, but it is unusual to find gross structural brain abnormalities. ${ }^{12}$

We report a patient with Moebius' syndrome who also has unilateral cerebellar hypoplasia.

\section{Case report}

A male infant was born at term by a normal vaginal delivery and his birth weight was $3250 \mathrm{~g}$. An amniocentesis had been performed during the pregnancy owing to advanced maternal age, but the pregnancy was otherwise uneventful. In the neonatal period he was noticed to have a right convergent squint and right sided facial weakness, although he breast fed without difficulty.

$\mathrm{He}$ smiled at 10 weeks and from five months he was able to roll over from the prone to the supine position. He could pass objects from one hand to the other by six months, although his mother noticed that left hand preference had already become established and there were occasional jerky movements of his right arm.

When reviewed at seven months there had been no change in his condition. His head circumference $(44.3 \mathrm{~cm})$, weight $(7.8 \mathrm{~kg})$, and length $(69 \mathrm{~cm})$ were 


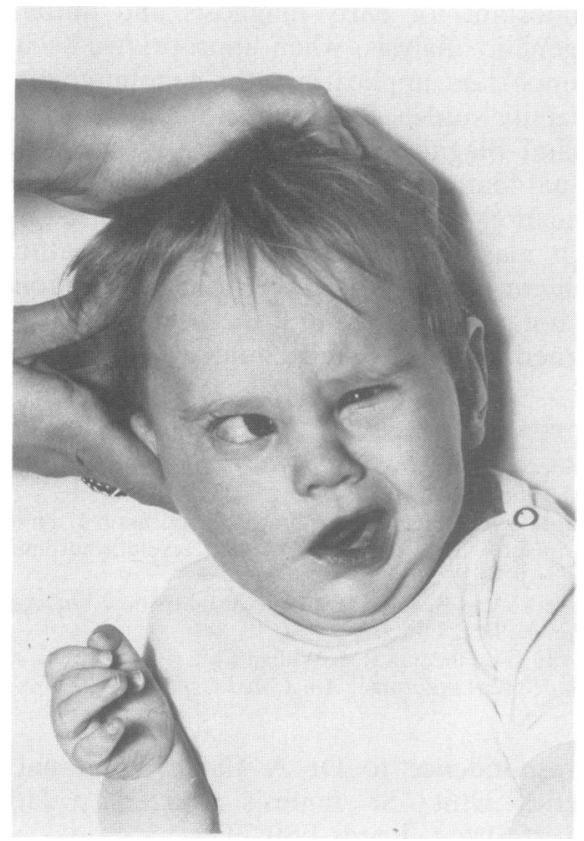

FIG 1 The patient at seven months of age showing complete paralysis of abduction of the right eye and right facial weakness.

between the 25th and 50th centiles. There was a bilateral convergent strabismus with complete paralysis of the right lateral rectus muscle and partial paralysis of the left. No movement of the right side of the face was apparent when smiling or crying (fig 1), but the left side of the face was normal. He was able to follow an object in a vertical trajectory without difficulty. Ocular fundi, pupil reactions, corneal reflexes, gag reflexes, and responses to sound were normal and there were no other abnormalities of the cranial nerves. He had no skeletal asymmetry or hypoplasia of peripheral muscles. Truncal tone, limb tone, and tendon reflexes were normal, although he was unable to sit unsupported. Slight ataxia of the right arm was apparent when reaching out for an object.

\section{IN VESTIGATIONS}

A CT scan showed hypoplasia of the right cerebellar hemisphere. This was further investigated by a magnetic resonance image (MRI) scan (figs 2 and 3) which indicated marked hypoplasia of the right cerebellar hemisphere, but the superior vermis was intact. The brain stem was small and rotated, the right half being smaller than the left. This is the result, presumably, of absence of ipsilateral corticocerebellar and spinocerebellar tracts. The left cerebellar hemisphere was morphologically normal and myelination was appropriate for age. No abnormality was shown above the tentorium.

The brain stem auditory evoked responses were normal on the left side but on the right side there was a markedly prolonged interval between waves and $\mathrm{V}$ consistent with an abnormality of the auditory pathways on the right. The electroencephalogram and flash visual evoked responses were normal. A normal $46, \mathrm{XY}$ karyotype had been detected prenatally.

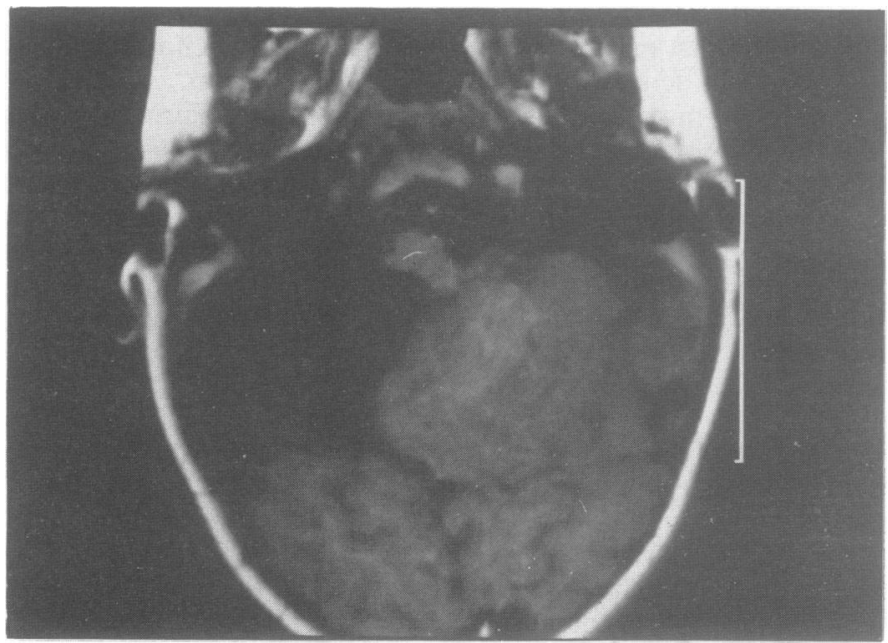

FIG 2 Axial image at the level of the middle cerebellar peduncle. The brain stem is small and asymmetrical. (T1 weighted spin echo sequence; $T R / T E=500 / 15 \mathrm{~m} \mathrm{sec}$.) 


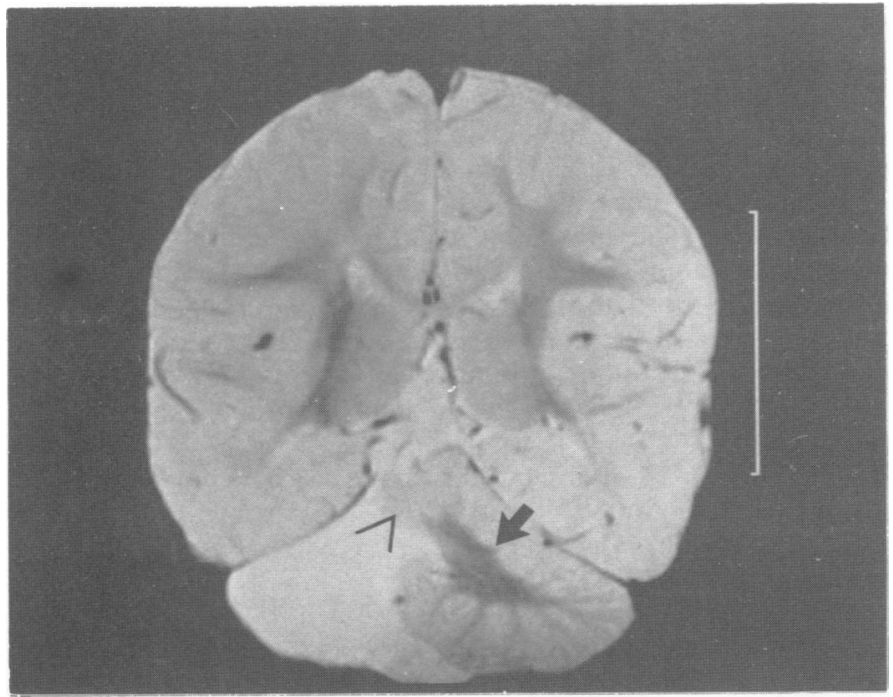

FIG 3 Coronal image at the level of the middle cerebellar peduncle (arrow) and thalami. Myelinated white matter appears dark. The left middle cerebellar peduncle is normally myelinated as is the rest of the cerebellar and cerebral white matter. The superior vermis (open arrow) is present but cerebrospinal fluid (white) fills the rest of the right cerebellar fossa. (STIR sequence $T R / T I / T E=3000 / 150 / 30 \mathrm{~m} \mathrm{sec}$.)

\section{Discussion}

We consider that the combination of unilateral cerebellar hypoplasia with Moebius' syndrome may be secondary to a vascular disruption involving the basilar artery, which occurred between 33 and 40 days of gestation. In the normal fetus the basilar artery begins forming between 29 and 33 days of gestation from fusion of the primitive longitudinal neural arteries, but the caudal portion of this fusion retains its plexiform character for some time. ${ }^{34}$

The longitudinal neural arteries are supplied mainly by the trigeminal arteries, which are branches of the internal carotid arteries. ${ }^{4}$ It is not until after about 54 days of gestation that the vertebral arteries become the major contributor of blood to the hindbrain, ${ }^{4}$ so it is possible that any impairment of flow in the basilar artery early in its formation may result in ischaemic disruption of the developing brainstem. Specifically, compromised vascular flow in the right longitudinal neural artery at the time of fusion with the left longitudinal neural artery could impair formation of the right cerebellar hemisphere. In addition, impaired circulation in the evolving right anterior inferior cerebellar artery and paramedian branches of the basilar artery could also affect the seventh and sixth cranial nerve nuclei respectively. Disruption of the posterior inferior cerebellar arteries has been associated with absence of the inferior vermis. ${ }^{5}$

Although the superior cerebellar artery arises from the cephalic end of the basilar artery at an earlier stage ( 33 days), it initially only supplies the vermis and caudal end of the tectum (future inferior colliculus), and hemispheric branches do not appear until later. ${ }^{4}$ In our patient the superior vermis was intact suggesting that normal vascular development had occurred before 33 days.

Although definitive evidence of a vascular disruption depends on histopathological examination or vertebral angiography, studies on other patients with Moebius' syndrome have suggested that some form of vascular disruption can occur. An arteriovenous malformation involving the lower pons and medulla has been described in association with extensive necrosis and gliosis of the brain stem and clinical findings of absent or impaired function of cranial nerves V to IX bilaterally. ${ }^{6}$

Bouwes-Bavinck and Weaver ${ }^{3}$ proposed that a vascular disruption in the subclavian artery distribution occurring around 42 days could result in a spectrum of abnormalities including Moebius' syndrome, Poland's, Klippel-Feil, or Sprengel's anomalies, isolated terminal transverse limb defects, or absence of the pectoralis major with breast hypoplasia. Combinations of these defects are well recognised and it has been estimated that in $15 \%$ of cases with Moebius' syndrome there are absent muscle groups, particularly of the pectoral or trapezius muscles. 'It may not be entirely appropriate to include Moebius' syndrome as part of the subclavian artery disruption sequence because most of the blood flow to the hindbrain early in development is from the internal carotid artery.

Vascular disruption has also been implicated in abnormalities of cerebellar development. Pascual- 
Castroviejo et $\mathrm{al}^{5}$ reported bilateral absence of the posterior inferior cerebellar arteries, and persistence of a left sided hypoglossal artery (shown by angiography) in a five year old girl with absent cerebellar tonsils and hypoplasia of the inferior cerebellar vermis. Diebler and Dulac $^{7}$ found porencephalic cysts in the parieto-occipital region in three patients with unilateral cerebellar hypoplasia and suggested that this was consistent with vascular obstruction in the territory of the basilar artery.

A patient with Moebius' syndrome and moderate unilateral cerebellar hypoplasia has been reported previously by Pitner et al. ${ }^{8}$ The child had facial diplegia, inability to abduct either eye, absent right corneal reflex, poor bulbar function, and atrophy of the right side of the tongue. At necropsy there was moderate hypoplasia of the right cerebellar hemisphere with an absent left inferior olivary nucleus. No atrophy of cranial nerve nuclei or nerves was found and the clinical condition was attributed to a primary muscle defect, as facial muscles showed moderate fibrosis with severe adipose replacement. The association of central nervous system pathology and a muscle defect was considered fortuitous. However, it is highly unlikely that hemiatrophy of the tongue could result from a primary myopathy. In addition the muscle pathology as described was also consistent with a neurogenic lesion, so that it is more likely that there was a neurogenic aetiology in this case. No abnormality was found in the vessels of the circle of Willis but there was no description of the smaller branches of the basilar artery.

Not all cases of Moebius' syndrome are considered to be the result of abnormalities of embryonal development and Towfighi, ${ }^{9}$ after reviewing 15 published necropsied cases, suggested some other contributing aetiologies: necrosis of previously normal cranial nerve nuclei owing to trauma at delivery, intrauterine viral infections, perinatal hypoxia, and primary muscle disease. The vulnerability of the immature brain stem to vascular impairment is recognised and at necropsy there is often more extensive necrosis of the brain stem than was suggested by clinical findings. ${ }^{10}$

Thus, Moebius' syndrome is a clinical entity with diverse aetiologies. In general, recurrence risks are small $(2 \%){ }^{1}$ The exceptions to this have been provided by three unusual families with a dominantly inherited syndrome. The family reported by Ziter et $a l^{11}$ had facial diplegia and finger contractures without ophthalmoplegia or limb hypoplasia. In each affected person there was an identical translocation between chromosomes 1 and 13 . Wishnick et al ${ }^{12}$ reported six subjects in two generations who had VI or VII nerve palsies, or both, plus limb abnormalities. A further nine members of this family had limb abnormalities alone. In the third family one child had bilateral VI and VII nerve palsies while her sib had isolated oligosyndactyly. ${ }^{13}$ The children's mother had slight facial weakness but no ophthalmoplegia.

The association of Moebius' syndrome with unilateral cerebellar hypoplasia is unusual but consistent with a vascular disruption occurring in the basilar artery early in its development. Vascular disruption should always be considered as one possible cause of Moebius' syndrome.

Dr M Hall-Craggs is a Research Fellow supported by Action Research for the Crippled Child. We thank Miss Jo Bramfitt for typing the manuscript.

\section{References}

${ }^{1}$ Baraitser M. Genetics of Moebius syndrome. J Med Genet 1977;114:415-7.

2 Sudarshan A, Goldie W. The spectrum of congenital facial diplegia (Moebius syndrome). Pediatr Neurol 1985;1:180-4.

${ }^{3}$ Bouwes-Bavinck JN, Weaver D. Subclavian artery supply disruption sequence: hypothesis of a vascular etiology for Poland, Klippel-Feil and Moebius anomalies. Am J Med Genet 1986;23:903-18.

4 Gillilan LA. Anatomy and embryology of the arterial system of the brain stem and cerebellum. In: Vinken PJ, Bruyn GW, eds. Handbook of clinical neurology. Vol 30. Amsterdam: North Holland, 1972:24-44.

5 Pascual-Castroviejo I, Tendero A, Martineze-Bermejo A, Lopez-Terradas JM, Casas CC. Persistence of the hypoglossal artery and partial agenesis of the cerebellum. Neuropediatrics 1975;6:184-9.

${ }^{6}$ Carter R, Waddy H, Manson J. Moebius syndrome. Abstract of paper presented at the Aust and NZ Society of Neuropathology Meeting, Adelaide, South Australia, 9 October 1987.

7 Diebler C, Dulac O. Cerebral and cranial malformations. In: Diebler C, Dulac O, eds. Pediatric neurology and neuroradiology. Vol 1. Berlin: Springer-Verlag, 1987:44-50.

8 Pitner SE, Edwards JE, McCormick WF. Observations on the pathology of the Moebius syndrome. J Neurol Neurosurg Psychiatry 1965;28:362-74.

9 Towfighi J, Marks K, Palmer E, Vannucci R. Moebius syndrome. Neuropathologic observations. Acta Neuropathol (Berl) $1979 ; 48: 11-17$

10 Thakkar N, O'Neil W, Duvally J, Liu C, Ambler M. Moebius syndrome due to brain stem tegmental necrosis. Arch Neurol 1977;34:124-6.

1 Ziter FA, Wiser WC, Robinson A. Three-generation pedigree of a Moebius syndrome variant with chromosome translocation. Arch Neurol 1977;34:437-42.

12 Wishnick MM, Nelson LB, Huppert L, et al. Moebius syndrome and limb abnormalities with dominant inheritance. Ophthalmic Paediatr Genet 1983;2:77-81.

${ }^{13}$ Mitter NS, Chudley AE. Facial weakness and oligosyndactyly: ?independent variable features of familial type of Moebius syndrome. Clin Genet 1983;24:350-4.

Correspondence to Dr M Baraitser, Department of Clinical Genetics, Institute of Child Health, 30 Guilford Street, London WC1N 1EH. 